\title{
Management of Limited Interocclusal Space Using UCLA Abutment to Retain the Prosthesis - A Case Report
}

\section{Shanta Chopra ${ }^{1 *}$, Sadhika $^{2}$, Manisha ${ }^{2}$ and Jasleen Arora ${ }^{3}$}

${ }^{1}$ MDS Prosthodontics, Senior Lecturer, Department of Prosthodontics, Genesis

Institute of Dental Sciences and Research, Ferozepur, Punjab, India

${ }^{2}$ MDS, Pedodontics Senior Lecturer, Department of Pedodontics and Preventive

Dentistry, Genesis Institute of Dental Sciences and Research, Ferozepur, Punjab,

India

${ }^{3}$ MDS Prosthodontics, Crown and Bridge and Oral Implantology, India

*Corresponding Author: Shanta Chopra, MDS Prosthodontics, Senior Lecturer,

Department of Prosthodontics, Genesis Institute of Dental Sciences and Research,

Ferozepur, Punjab, India.
Received: October 29, 2021

Published: November 25, 2021

(C) All rights are reserved by Shanta Chopra., et al.

\begin{abstract}
Using either cement or screw to retain prosthesis depends on the clinician's choice. Both mode of treatment has their own advantages and disadvantages. Cement retained has better esthetics, better access, and low cost whereas screw-retained prosthesis preserves gingival health and its predictable retrievability that can be achieved without damaging the restoration or fixture. This case report presents a case with the replacement of mandibular molar due to inadequate interocclusal space for cement retained prosthesis and the situation was managed with UCLA abutment.
\end{abstract}

Keyword: Screw Retained; Cement Retained; Esthetics; Retrievability; Inadequate Interocclusal Space; UCLA

\section{Introduction}

Loss of a tooth/teeth may cause functional and esthetic deficits to the patient. The common reason for tooth loss in the posterior region includes-Dental caries, Periodontal diseases, Congenital missing teeth. If tooth loss is not rehabilitated can lead to migration of teeth which impinge the space making it highly unesthetic especially in the anterior region and responsible for food lodgment in the posterior region. So, rehabilitation of lost teeth is very essential. This rehabilitation can be done with both removable as well as fixed methods.

Rehabilitation of the completely or partially edentulous patient with implants has offered promising results. Implant prosthesis improves mastication which ultimately makes patients more confident. Implant prosthesis can be retained using both cement or screw. Screw retained prosthesis are indicated in cases with mal-aligned implants, limited interarch space (less than $5 \mathrm{~mm}$ ) to keep adequate abutment height, provisional restorations, in full mouth rehabilitation cases or in cases where implants are deeply placed [1]. Whenever there is a need to repair the restoration the prosthesis may get damaged when the cement seal cannot break off easily. So, prosthesis retained with screw is beneficial in protecting both prosthesis as well as the gingival health by preventing inflammation caused by cement in gingival sulcus. Assessment of surrounding tissue, screw replacement is easy and it required only radiographic verification. According to Misch, the success rate for a single implant is $97 \%$ for 10 years [2]. The long-term durability and success of these implant prosthesis depend on the precise fit and maintenance of the prosthesis.

\section{Case Report}

A 57-year-old male reported with the chief complaint of difficulty in chewing food due to missing teeth. Upon intra-oral examination, the left mandibular first molar was found to be miss- 
ing. It was observed that patient had limited interocclusal space of less than $5 \mathrm{~mm}$. however, the mesio-distal width and bucco-lingual width was adequate. So, it was decided to continue the prosthetic phase with screw retained prosthesis. Detailed dental and medical history was recorded but nothing relevant was reported. It was decided for implant placement in region.

Different treatment options were given to the patient:

- A Conventional removable partial denture

- $\quad$ Cast Partial denture

- $\quad$ Essix appliance

- A Conventional fixed partial denture

- Resin Bonded fixed partial denture

- $\quad$ Single Implant placement.

But using the conventional removable partial denture can be very galling as it compromised aesthetics and is not at all comfortable to be patient. Cast partial dentures display metal which is again highly unesthetic and requires tooth preparation of the abutment teeth. Essix appliance is also another treatment option but is not recommended for long use due to rapid wear [3]. It is useful as a provisional restoration.

Using Conventional fixed partial denture is also not considered because it requires massive tooth preparation and hence is not conservative. Resin-bonded fixed partial denture such as Maryland Bridge require fully erupted tooth to provide sufficient enamel to retain the prosthesis, its retention depends upon the strength of metal used and is not indicated for long-term service.

The use of dental implants is a standard and treatment of choice as it is more conservative as well as esthetic.

The patient had given his consent for a dental implant prosthesis. After discussing the pros and cons of each treatment option, it was decided to rehabilitate the missing tooth with a Single Implant placement.

- A diagnosis was done on OPG to check for a surgical site for placement of the implant.

- $\quad$ According to OPG Implant length $11.5 \mathrm{~mm}$ and diameter $4.2 \mathrm{~mm}$ of Noris Medical implant was planned followed by delayed loading protocol to have better implant stability.
- The patient was prepared for the first phase of treatment. The implant was placed under Local Anesthesia by following a strict protocol of sterilization and disinfection.

- The initial osteotomy was done with a pilot drill and the site was enlarged in width and depth of $11 \mathrm{~mm}$.

- Once the implant site was prepared, the implant was placed (Noris Medical $4.2 \times 11.5 \mathrm{~mm}$ implant)

- The implant was tightened to $45 \mathrm{~N} \mathrm{~cm}$, exhibiting good primary stability.

- $\quad$ The cover screw was placed and the surgical site was sutured with 3-0 vicryl resorbable sutures.

- Immediate post-operative OPG was taken to confirm the final position of the implant placed and post-operative instructions were given to the patient (Figure 2).

- The patient was recalled after 3 months. Under local anesthesia, the mucoperiosteal flap was raised and a healing abutment was placed (Figure 3).

- The patient was recalled after 1 week. This is to enhance healthy gingival biotype to enhance good gingival collar or emergence profile of the restoration (Figure 4).

- Impression coping was inserted, the correct angulation was seen and it was decided to use UCLA abutment with internal hex. (Plastic Castable Abutment Hex, Noris Medical) (Figure 5).

- Fixture level impression was made with Polyvinylsiloxane (Putty and Light body consistency) impression material (Flexceed, GC) (Figure 6) and soft tissue gingival mask (Esthetic Mask, Detax) was applied.

- The impression was disinfected with $2 \%$ glutaldehyde and the master cast was poured in type IV gypsum product (Kalabhai, Ultrarock) that incorporate a soft tissue gingival mask (Figure 7).

- $\quad$ Cast retrieved with transfer coping. This coping is replaced with plastic castable abutment UCLA.

- This plastic sleeve or UCLA abutment was incorporated into the wax pattern with inlay wax (Bego, Germany) for metal ceramic restoration was fabricated. The UCLA abutment was trimmed to such an extend such as to obtain sufficient height for the crown replacement at the same time ensuring no high points. (Figure 8). 
- Wax pattern and cast in Nickel Chromium base metal alloy (Bego, Germany) (Figure 9).

- Metal try-in was done and the marginal fit of the prosthesis was evaluated.

- $\quad$ Porcelain firing (Vita Company, Germany) with appropriate shade was done, evaluated for fit and required adjustment was done (Figure 10).

- The retaining screw was placed and threaded through the crown. Proper abutment seating onto the implant fixture was verified radiographically (Figure 11).

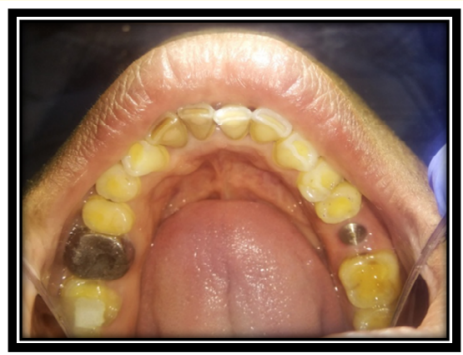

Figure 3: Healing abutment placed.

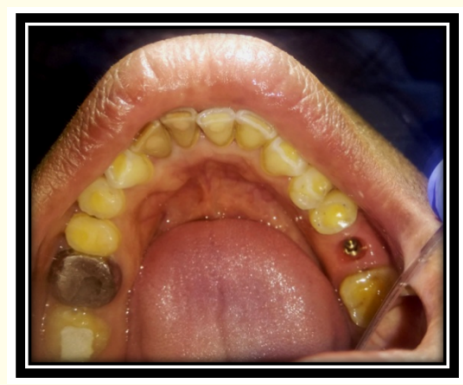

Figure 4: Gingival collar formation.

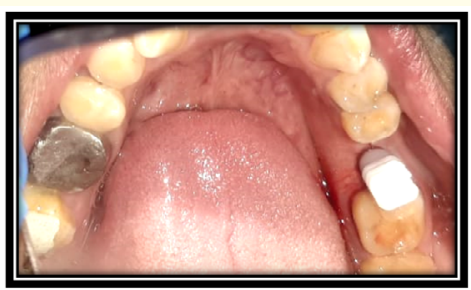

Figure 5: Impression coping placed.

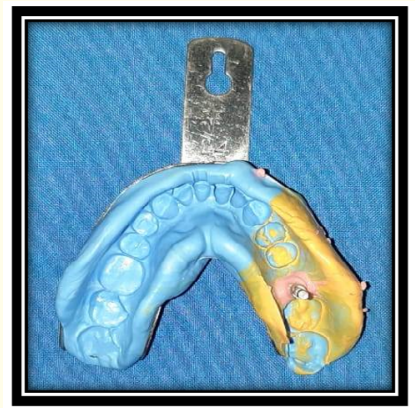

Figure 2: Post-Operative OPG. 


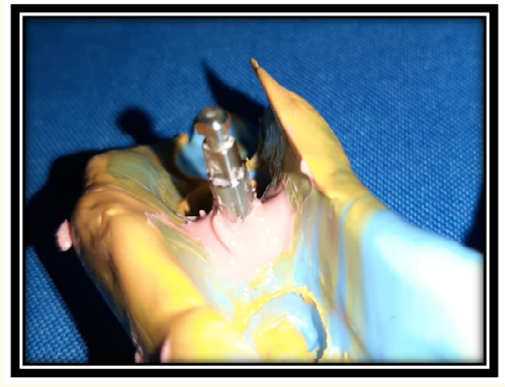

Figure 6: Impression made with addition silicone (putty and light body consistency) and Gingival mask applied.
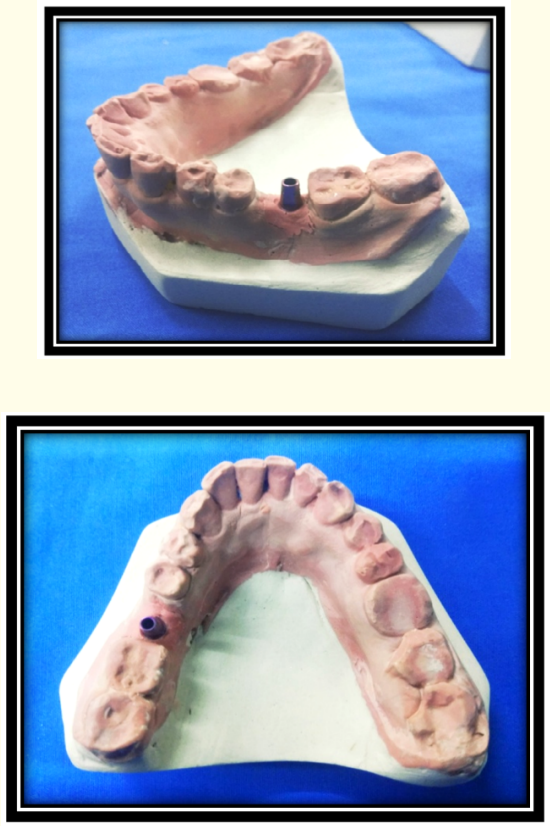

Figure 7: Master cast retrieved with transfer coping.

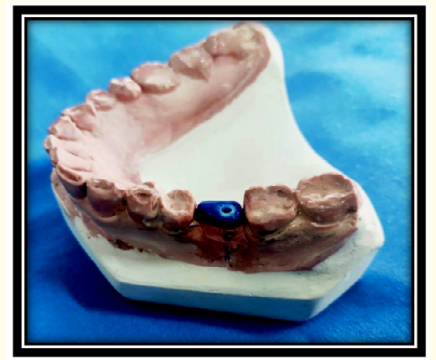

Figure 8: Wax pattern for metal ceramic crown.

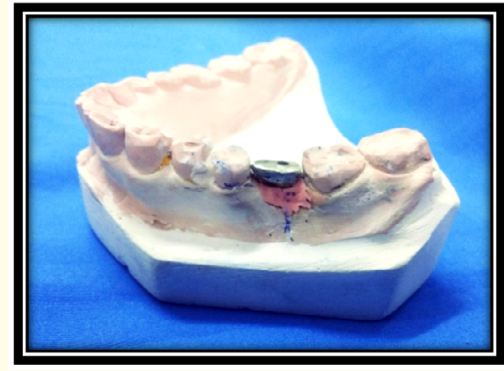

Figure 9: Metal crown fabrication.

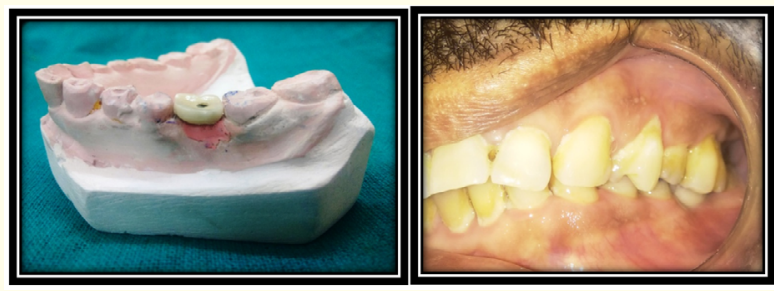

Figure 10: Porcelain fused metal crown fabrication and evaluated intaorally.

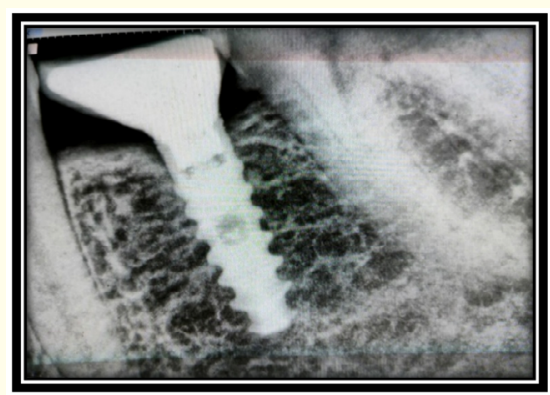

Figure 11: Post cementation IOPAR to evaluate complete seating of the prosthesis.

\section{Discussion}

A dental implant consists of three parts- Fixture or the root part, Abutment intermediate between crown and fixture, and the Prosthesis. An abutment is defined as a part of a structure that directly receives thrust or pressure. It is a tooth, a portion of a tooth, or that portion of a dental implant that serves to support and/or retain a prosthesis (GPT-9) [4]. These abutments can be of various 
types such as prefabricated or customized abutment, with internal or external connection, cement or screw retained abutment.

UCLA (Universal Castable Long Abutment) [5] type of abutment directly attaches to the implant and provide a pattern for fabrication of screw retained restoration [6]. These are basically custom-made abutment designed for a single implant. It lacks anti-rotational elements. They are available in various forms such as traditional plastic form, gold form, plastic sleeve with titanium base, plastic sleeve with cobalt chromium sleeve, gold base with plastic sleeve. They are indicated in single implants, in limited interocclusal space, or in cases of tilted implants. It requires a highly accurate impression of the implant. These have been used over the past 25 years in the restoration of osseointegrated implants. They have a subgingival margin allowing them to be used in minimal interocclusal clearance [7].

When interarch occlusal space is limited, clinician tend to avoid cement retained prosthesis. This is because preferring cement retained prosthesis leads to overreduction of abutment height thus affecting the retention of the prosthesis as it will dislodged easily. In these cases, screw retained prosthesis as UCLA abutments are preferred. They are attached directly to implant without ant intervening abutment [8]. They have a plastic burnout pattern. It consists of a large access hole in the centre which is the place for screw and a short length collar gingivally. Screw used can be made of titanium or gold [8].

Cement retained implant prosthesis are easy to fabricate, provide superior esthetics and optimal occlusal design whereas screw retained prosthesis are more advantageous as they are easy to retrieve, protect gingival health as no cement is used, and ease of hygiene maintenance (Wittneben., et al. 2000) [9]. But mechanical complications are more common in screw retained prosthesis such as screw loosening followed by accumulation of a granulation tissues leading to fistulae formation and plaque deposition resulting in screw fracture. Moreover, the chance of loss of retention for screw retained restoration is high about $65 \%$ and cement retained is as low as $5 \%[1]$.

\section{Conclusion}

Implant prosthesis can be retained both Cement Retained or Screw Retained. Both modes of rehabilitation have pros and cons. Using screw retained prosthesis helps in easy retrievability of the prosthesis. However, it has a major complication of screw loosing due to biomechanical overloading. To avoid this problem regular follow-up, need to give to the patient. UCLA abutments can be the best treatment in patients with limited interarch space. Adequate treatment planning, knowledge of the occlusal scheme, tightening to the correct torque, and regular recall appointments will minimize the incidence of abutment screw loosening and fracture.

\section{Bibliography}

1. Rola Shadid and Nasrin Sadaqa. "A Comparison Between Screw- and Cement-Retained Implant Prostheses. A Literature Review". Journal of Oral Implantology 38.3 (2012): 298-307.

2. Misch CE. "The importance of dental implants". General Dentistry 49 (2001): 38-45.

3. Chopra S., et al. "Essix Appliance: An Innovation Modification for use as Temporary Bridge-A Case Report". Journal of Advanced Medical and Dental Sciences Research 8.1 (2020): 184-186.

4. "The Glossary of Prosthodontic terms". Journal of Prosthetic Dentistry 117.5S (2017).

5. Goiato MC., et al. "Dynamic UCLA for single tilted implant in an aesthetic region". International Journal of Surgery Case Reports 7 (2015): 149-153.

6. Singh N and Jatana CS. "Correction of misaligned implantsUCLA abutment". Journal of Interdisciplinary Dentistry 4.3 (2014): 156.

7. Sorensen J., et al. "Nobelpharma vs UCLA abutment components: Vertical and horizontal interface fidelity". In Palm Springs, Calif: UCLA Implant Symposium (1990).

8. Prisco R., et al. "New abutment for a screw-retained, implantsupported crown". The Journal of Prosthetic Dentistry 85.1 (2001): 30-33.

9. Wittneben JG., et al. "Screw retained vs. cement retained implant-supported fixed dental prosthesis". Periodontology 73.1(2000): 141-151.

\section{Volume 5 Issue 12 December 2021 (C) All rights are reserved by Shanta Chopra., et al.}

